\title{
Analysis of blood neutrophil elastase, glutathione levels and pathological findings in postoperative acute exacerbation of idiopathic pulmonary fibrosis associated with lung cancer: Two case reports
}

\author{
KEISHI SUGINO ${ }^{1}$, YASUHIKO NAKAMURA ${ }^{1}$, YOKO MURAMATSU ${ }^{1}$, \\ YOSHINOBU HATA ${ }^{2}$, KAZUTOSHI SHIBUYA ${ }^{3}$ and SAKAE HOMMA ${ }^{1}$ \\ Departments of ${ }^{1}$ Respiratory Medicine, ${ }^{2}$ Chest Surgery and ${ }^{3}$ Surgical Pathology, \\ Toho University School of Medicine, Ota-ku, Tokyo 143-8541, Japan
}

Received May 27, 2016; Accepted July 25, 2016

DOI: $10.3892 /$ mco.2016.993

\begin{abstract}
Acute exacerbation (AE) of idiopathic pulmonary fibrosis (IPF) is characterized by severe worsening dyspnea and high mortality. It has been proven that the serum neutrophil elastase (NE) level, in addition to the serum Krebs von den Lungen-6 (KL-6) and surfactant protein-D (SP-D) levels, was elevated in patients with IPF-AE. Glutathione (GSH) is the major antioxidant involved in cell metabolism and survival. It is also known that IPF is characterized by reduced GSH levels in bronchoalveolar lavage fluid and blood. Case 1 was a 67-year-old man who was referred to our hospital complaining of a 2-year history of progressive dyspnea on exertion (DOE). The patient was initially diagnosed with IPF, followed by inhaled $\mathrm{N}$-acetylcysteine monotherapy. Two years later, left upper lobectomy with lymph node dissection was performed due to primary lung cancer, which was large-cell neuroendocrine carcinoma (pT2aN2M0, stage IIIA). Five days after lung surgery, the patient developed AE. Case 2
\end{abstract}

Correspondence to: Dr Keishi Sugino, Department of Respiratory Medicine, Toho University School of Medicine, 6-11-1 Omori-nishi, Ota-ku, Tokyo 143-8541, Japan

E-mail:ks142129_ikusou@ybb.ne.jp

Abbreviations: AE, acute exacerbation; IPF, idiopathic pulmonary fibrosis; NE, neutrophil elastase; GSH, glutathione; rGSH, reduced glutathione; GSSG, oxidized glutathione; DOE, dyspnea on exertion; UIP, usual interstitial pneumonia; DAD, diffuse alveolar damage; ARDS, acute respiratory distress syndrome; NAC, N-acetylcysteine; HRCT, high-resolution computed tomography; PFT, pulmonary function test; VC, vital capacity; DLCO, diffusing capacity of the lungs for carbon monoxide; GGO, ground-glass opacities; KL-6, Krebs von den Lungen-6; SP-D, surfactant protein-D; DHP-PMX, direct hemoperfusion with an immobilized polymyxin B column

Key words: idiopathic pulmonary fibrosis, acute exacerbation, lung cancer, neutrophil elastase, glutathione was a 67-year-old man who was referred to our hospital with suspected lung cancer, complaining of dry cough and DOE. The patient underwent left upper lobectomy with lymph node dissection for primary lung cancer, which was diagnosed as well-differentiated adenocarcinoma (pT2aN2M0, stage IIIA). Ten days after lung surgery, the patient developed AE. The levels of biomarkers, such as serum NE, redox balance [reduced GSH (rGSH)/oxidized GSH (GSSG)] in the blood, as well as the correlation between serial changes of these biomarkers and prognosis, were analyzed in 2 patients with postoperative IPF-AE associated with lung cancer. Interestingly, the serial changes of the serum rGSH/GSSG ratio may suggest the possibility of predicting the onset of postoperative AE and/or survival, along with serum NE levels.

\section{Introduction}

Idiopathic pulmonary fibrosis (IPF) is a chronic, progressive fibrotic lung disease of unknown etiology, with a median survival from diagnosis of 3-5 years. The clinical course of individual patients with IPF is variable and unpredictable (1). In particular, although Collard et al (2) proposed the definition of IPF-acute exacerbation (AE) in 2007, the etiology of IPF-AE remains uncertain. However, there are some reports regarding the development of IPF-AE after surgical lung resection. Reported histological findings from lung biopsy in IPF-AE described not only the typical usual interstitial pneumonia (UIP) pattern, but also diffuse alveolar damage (DAD) (3). Several therapies for IPF-AE have been introduced thus far, but survival remains poor.

Neutrophil elastase (NE) is an elastolytic enzyme that is released from activated neutrophils and is known to involve lung injury, such as acute respiratory distress syndrome (ARDS) (4). Glutathione (GSH) is the major antioxidant involved in cell metabolism and survival (5). It is also known that IPF is characterized by GSH deficiency in bronchoalveolar lavage fluid (6). As described by Teramoto et al (7), the amount of oxidized GSH (GSSG) in the blood is significantly increased in patients with IPF. Moreover, Muramatsu et al (8) 
A

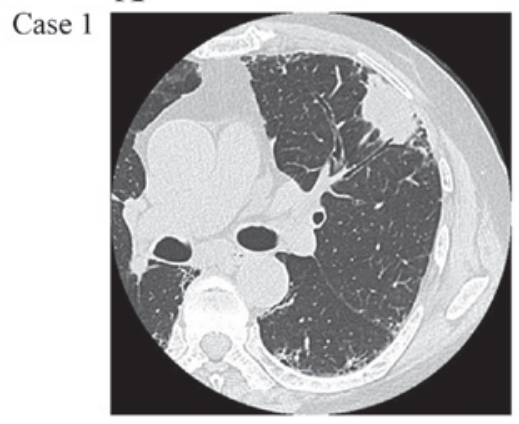

D

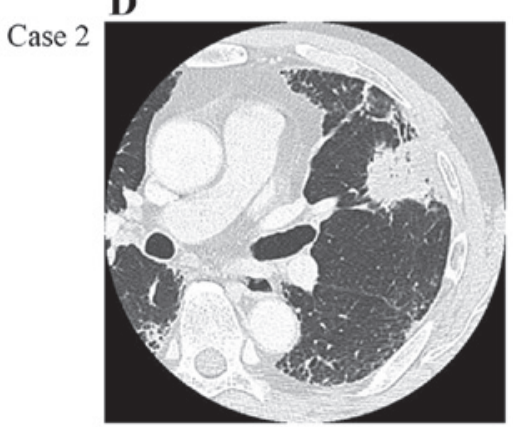

B

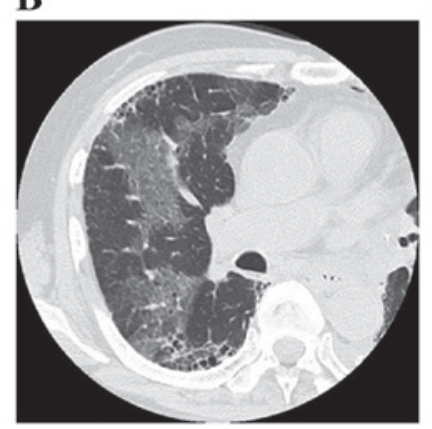

E

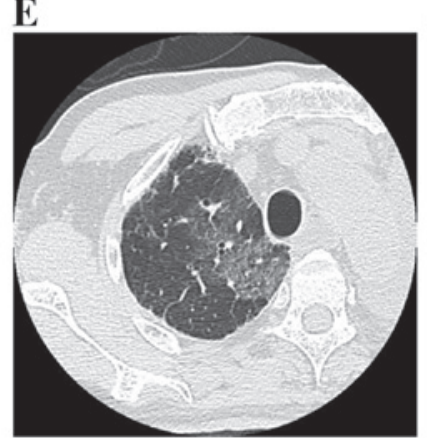

C

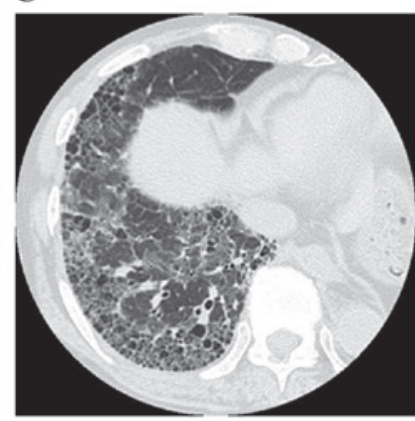

$\mathbf{F}$

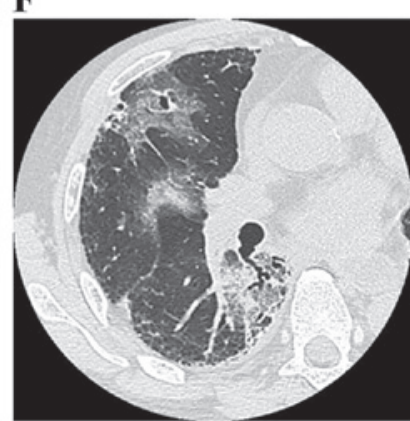

Figure 1. Chest high-resolution computed tomography (HRCT) images. Case 1: (A) Chest HRCT scan prior to surgery showing a lobulated subpleural mass in the left upper lobe, measuring $4 \mathrm{~cm}$ in diameter, on a background of reticulation and honeycombing, predominantly in the bilateral lower lobes. (B and C) Chest HRCT scans on day 5 after surgery showing multifocal ground-glass opacities (GGO), predominantly in the non-operated right lung. Case 2: (D) Chest HRCT scan prior to surgery showing a subpleural mass in the left upper lobe exhibiting spiculation and air bronchogram, measuring $7.5 \mathrm{~cm}$ in diameter, with reticulation and faint honeycombing, predominantly in the bilateral lower lobes (usual interstitial pneumonia pattern). (E and F) Chest HRCT scans on day 10 after surgery showing multifocal GGO predominantly in the non-operated right middle and lower lobes.

reported that inhaled $\mathrm{N}$-acetylcysteine (NAC) monotherapy was associated with improvement of the redox imbalance in patients with early IPF. Hence, we investigated whether novel biomarkers, such as serum NE and redox balance [reduced GSH (rGSH/GSSG)] were more rapid and highly sensitive indicators in the postoperative IPF-AE setting. These biomarkers were analyzed immediately prior to surgery, on the day of onset of IPF-AE, and 3 days after IPF-AE.

The present study was approved by the local Ethics Committee of the Toho University Omori Medical Center (no. 23-60; approval date, 30/09/2011).

\section{Case reports}

Case 1. A 67-year-old man was referred to our hospital complaining of a 2-year history of progressive dyspnea on exertion (DOE). The patient was initially diagnosed with IPF and received inhaled NAC monotherapy. Two years later, chest high-resolution computed tomography (HRCT) scans revealed a $4-\mathrm{cm}$ lobulated subpleural mass in the left upper lobe on a background of reticulation, with honeycombing, predominantly in the bilateral lower lobes (Fig. 1A-C). Pulmonary function tests (PFT) revealed a vital capacity (VC) of 2.731 (79.4\% of predicted value), with decreased diffusing capacity of the lungs for carbon monoxide (DLCO; $53.7 \%$ of predicted value). The severity of IPF was stage I in accordance with the Japanese Respiratory Society criteria (9). Left upper lobectomy with lymph node dissection was performed (operative time, 161 min; mean $\mathrm{PaO}_{2}$ during operation, 89.5 Torr; intraoperative blood loss, $50 \mathrm{ml}$ ).
On postoperative histological examination, there was heterogeneous interstitial fibrosis with honeycombing in a subpleural and perilobular distribution pattern, alternating with areas of normal lung tissue. Furthermore, fibroblastic foci were sporadically present in a dense collagen fibrosis background, with infiltration by abundant lymphocytes and neutrophils (Fig. 2A and B). Invasive large-cell neuroendocrine carcinoma adjacent to the honeycomb lesions was diagnosed (pT2aN2M0, stage IIIA). Five days after lung surgery, the patient complained of dyspnea with acutely decreased oxygenation $\left(\mathrm{PaO}_{2} / \mathrm{FiO}_{2}=215\right)$. Chest HRCT scans revealed multifocal ground-glass opacities (GGO), predominantly in the non-operated right lung (Fig. 1B and C). In addition, the levels of serum NE and GSSG were significantly increased, with a decreased $\mathrm{rGSH} / \mathrm{GSSG}$ ratio. However, the levels of serum Krebs von den Lungen-6 (KL-6) and surfactant protein-D (SP-D) remained unchanged (Fig. 3). After the patient was diagnosed with AE, he was treated with methylprednisolone (1,000 mg/day) intravenously for 3 days, followed by a tapered dose based on his respiratory condition. At the same time, the patient received a synthetic NE inhibitor, recombinant human soluble thrombomodulin, cyclosporine A and pirfenidone. Furthermore, direct hemoperfusion with an immobilized polymyxin B column (DHP-PMX) was performed once daily ( $6 \mathrm{~h} / \mathrm{day}$ ) for 2 days. Three days after the initiation of these combination therapies, the patient's general condition had significantly improved, with an increase in the $\mathrm{PaO}_{2} / \mathrm{FiO}_{2}$ ratio from 215 to 355 . Moreover, the levels of serum NE and the rGSH /GSSG ratio immediately improved (Fig. 3). 
A

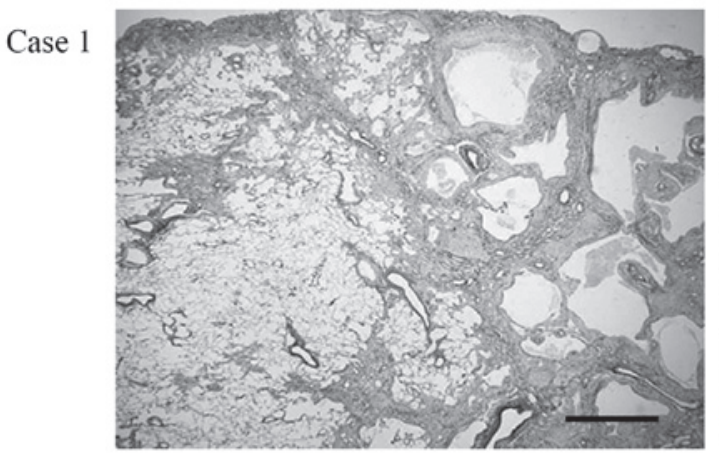

C

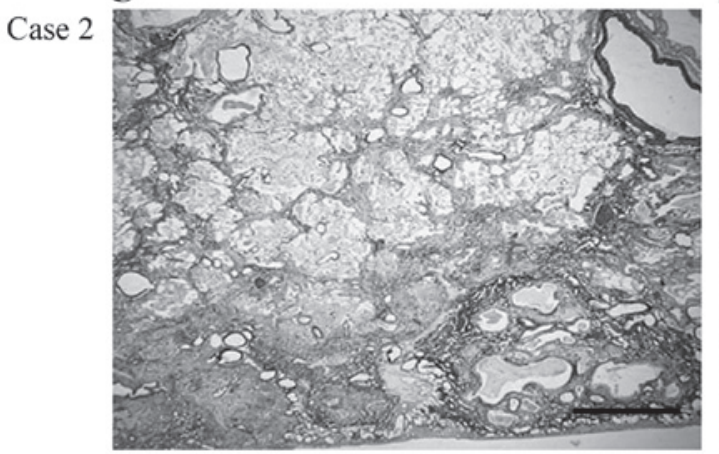

B

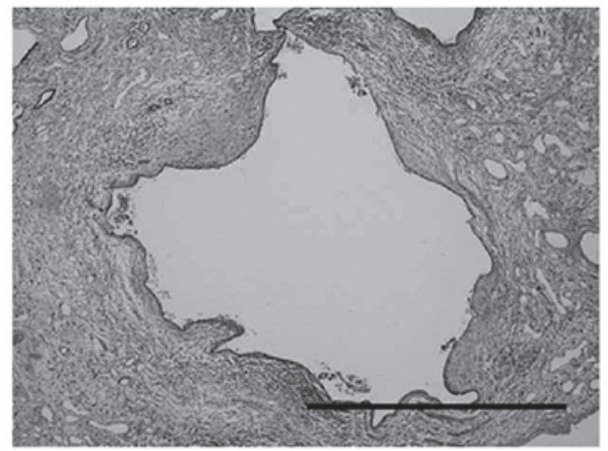

D

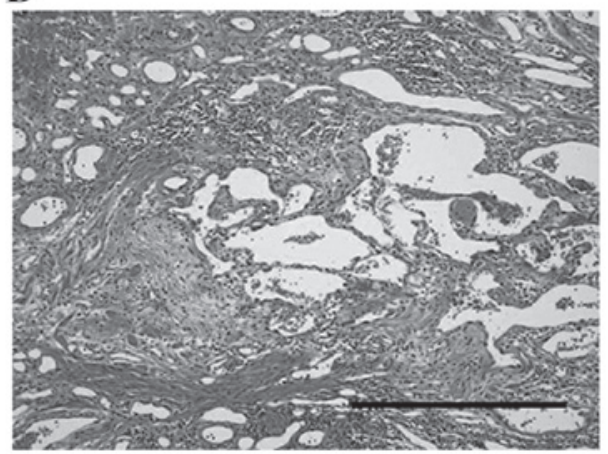

Figure 2. Microscopic appearance of the resected lungs. Case 1: (A) Heterogeneous interstitial fibrosis with honeycombing with a subpleural and perilobular distribution pattern, alternating with areas of normal lung tissue (Elastic van Gieson stain; scale bar, $1 \mathrm{~mm}$ ). (B) Fibroblastic foci are sporadically present in a dense collagen fibrosis background and infiltration by abundant lymphocytes and neutrophils is observed (hematoxylin and eosin stain; scale bar, 1 mm). Case 2: (C) Background pattern of usual interstitial pneumonia with fibrotic changes, predominantly distributed in the subpleural and perilobular areas, and an abrupt transition between almost-normal alveolar septa and dense fibrosis with architectural disruption (Elastic van Gieson stain; scale bar, 500 mm). (D) Prominent fibroblastic foci and infiltration by lymphocytes and neutrophils in the interstitium (hematoxylin and eosin stain; scale bar, 500 mm).

Case 2. A 67-year-old man was referred to our hospital with suspected lung cancer, complaining of dry cough and DOE. Chest HRCT scans showed a subpleural mass, sized $7.5 \mathrm{~cm}$, in the left upper lobe, exhibiting spiculation and air bronchogram, as well as reticulation and faint honeycombing, predominantly in the bilateral lower lobes (UIP pattern) (Fig. 1D-F). The PFT indicated restrictive impairment with decreased diffusion capacity, with a VC of 2.641 (74.6\% of predicted value) and a DLCO $61.7 \%$ of the predicted value. The patient was diagnosed with stage I IPF and underwent left upper lobectomy with lymph node dissection (operative time, $239 \mathrm{~min}$; mean intraoperative $\mathrm{PaO}_{2}, 387$ Torr; intraoperative blood loss, $160 \mathrm{ml})$.

The postoperative histological examination revealed a background pattern of UIP with fibrotic changes predominantly distributed in the subpleural and perilobular areas, and an abrupt transition between almost-normal alveolar septa and dense fibrosis with architectural disruption. Additionally, there were prominent fibroblastic foci and a lymphocyte and neutrophil infiltration of the interstitium (Fig. 2C and D). Well-differentiated adenocarcinoma was detected adjacent to the fibrotic lesions (pT2aN2M0, stage IIIA). Ten days after lung surgery, the patient complained of dyspnea and the oxygenation progressively worsened $\left(\mathrm{PaO}_{2} / \mathrm{FiO}_{2}=281\right)$. A chest HRCT scan revealed multifocal GGO, predominantly in the non-operated right middle and lower lobes (Fig. 1E and F). Similar to case 1 , the levels of serum NE were increased, with a decreased rGSH/GSSG ratio (Fig. 3). By contrast, the serum KL-6 and SP-D levels were decreased. The patient was treated with methylprednisolone $(1,000 \mathrm{mg} /$ day) intravenously for 3 days, followed by a tapered dose of $60 \mathrm{mg} / \mathrm{day}$. At the same time, a synthetic NE inhibitor was administered and DHP-PMX was performed once daily (6 h/day) for 2 days. Despite these treatments, the patient's general condition rapidly deteriorated, with the $\mathrm{PaO}_{2} / \mathrm{FiO}_{2}$ ratio decreasing from 281 to 111 . Additionally, the value of the $\mathrm{rGSH} / \mathrm{GSSG}$ ratio in the blood decreased, in addition to elevation of the serum NE, KL-6 and SP-D levels (Fig. 3). Four days after the onset of $\mathrm{AE}$, the patient succumbed to acute respiratory failure due to IPF-AE.

Written informed consent was obtained from the patients' next-of-kin for the publication of this manuscript and any accompanying images.

\section{Discussion}

The incidence of postoperative IPF-AE has been reported to range from 0 to $25 \%$, with a mortality rate of $33.3-100 \%$ (10). However, considering the high risk of IPF-AE following chemotherapy or radiotherapy, surgical lung resection is the first choice of treatment for IPF patients with early-stage lung cancer. Sakamoto et al (11) reported that possible inciting factors in postoperative IPF-AE may include the administration of oxygen supplementation at a high concentration and/or surgical stress, including mechanical ventilation-related lung injury. Padley et al (12) reported that ARDS following pulmo- 

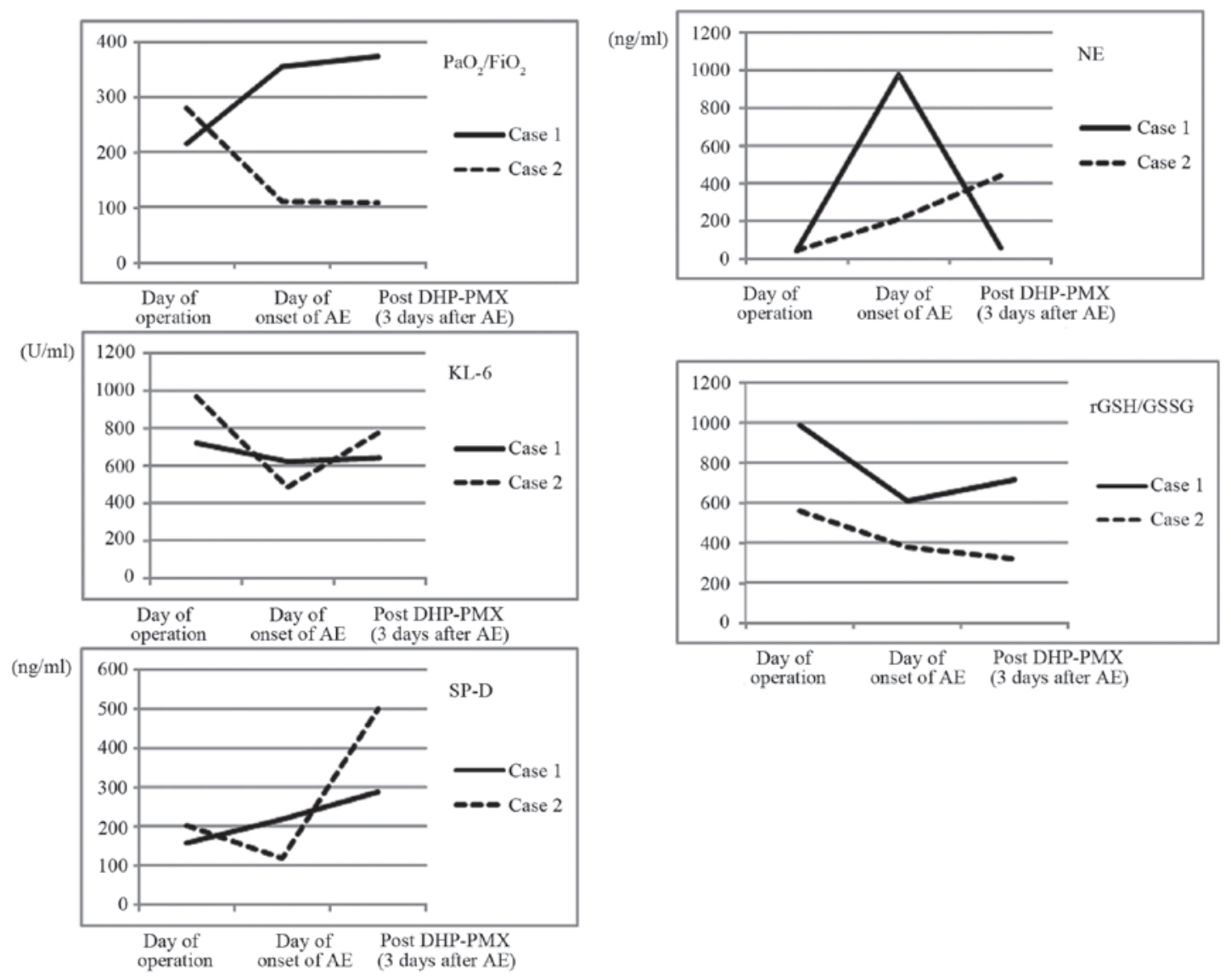

Figure 3. Changes in laboratory data before and after postoperative IPF-AE. IPF, idiopathic pulmonary fibrosis; AE, acute exacerbation; DHP-PMX, direct hemoperfusion with an immobilized polymyxin B column; KL-6: Krebs von den Lungen-6; SP-D: surfactant protein-D; NE, neutrophil elastase; GSH, glutathione; GSSG, oxidized GSH; rGSH, reduced GSH.

nary resection occurs mainly in the non-operated lung. In fact, intraoperative high concentrations of inspired oxygen may have triggered the IPF-AE in case 2. Furthermore, the present cases exhibited increased GGO on the non-surgical side receiving single-lung ventilation. These results suggest that hyperoxia, barotrauma, volutrauma and biotrauma may be involved in the DAD of IPF-AE.

IPF-AE histologically manifests as acute or organizing DAD, and less commonly as profuse organizing pneumonia superimposed on underlying UIP (3). Tiitto et al (13) reported that the number of fibroblastic foci (FF) in lung samples prior to death was associated with poor survival, but not with DAD of patients with UIP, suggesting that the number of FF cannot predict an IPF-AE. Although there were significant numbers of FF and infiltration by lymphocytes and neutrophils in fibrotic lesions in the cases presented herein, the outcomes after postoperative IPF-AE differed. These histological findings may indicate that both patients had highly active IPF.

$\mathrm{NE}$ is one of the most destructive enzymes, with the capability of degrading almost all extracellular matrix, and plays a crucial role in the pathophysiology of ARDS (4). Furthermore, it has been proven that the serum NE level, in addition to serum KL-6 and SP-D levels, was elevated in patients with IPF-AE (4). We hypothesized that the serial changes of the serum NE level may be associated with the onset of postoperative IPF-AE and survival. In addition, a marked deficiency of the major antioxidant GSH has been previously described in patients with IPF (6). Thus, administration of antioxidants such as NAC, a precursor of GSH, is a potential treatment option for IPF patients. Recently, Homma et al (14) reported the clinical efficacy of inhaled NAC monotherapy in patients with early-stage IPF. More recently, Muramatsu et al (8) reported that inhaled NAC monotherapy was associated with improved redox imbalance in patients with early IPF. NAC administration may contribute to the restoration of the serum GSH balance and improve the outcome after developing AE of IPF, as the patient in case 1 who achieved postoperative IPF-AE was treated with inhaled NAC monotherapy for 2 years. In the 2 cases reported herein, the serial change in the serum value of the $\mathrm{rGSH} / \mathrm{GSSG}$ ratio may suggest the possibility of predicting the onset of postoperative $\mathrm{AE}$ and/or survival, along with serum NE level.

To the best of our knowledge, this is the first study to evaluate the serial changes of GSH levels in the blood of patients with postoperative IPF-AE associated with lung cancer. Further studies are required to validate our methodologies and results.

\section{Acknowledgements}

The authors are grateful to J. Tatebe (Department of Clinical Laboratory, Toho University Omori Medical Center, Tokyo, Japan) for the advice and analysis of the patient's GSH and NE levels. This study was supported by the Practical Research 
Project for Rare Intractable Diseases from the Japan Agency for Medical Research and Development (AMED).

\section{References}

1. Raghu G, Collard HR, Egan JJ, Martinez FJ, Behr J, Brown KK, Colby TV, Cordier JF, Flaherty KR, Lasky JA, et al: An official ATS/ERS/JRS/ALAT statement: Idiopathic pulmonary fibrosis: Evidence-based guidelines for diagnosis and management. Am J Respir Crit Care Med 183: 788-824, 2011.

2. Collard HR, Moore BB, Flaherty KR, Brown KK, Kaner RJ King TE Jr, Lasky JA, Loyd JE, Noth I, Olman MA, et al: Acute exacerbations of idiopathic pulmonary fibrosis. Am J Respir Crit Care Med 176: 636-643, 2007.

3. Parambil JG, Myers JL and Ryu JH: Histopathologic features and outcome of patients with acute exacerbation of idiopathic pulmonary fibrosis undergoing surgical lung biopsy. Chest 128 : 3310-3315, 2005.

4. Kawabata K, Hagio T and Matsuoka S: The role of neutrophil elastase in acute lung injury. Eur J Pharmacol 451: 1-10, 2002.

5. Hunninghake GW: Antioxidant therapy for idiopathic pulmonary fibrosis. N Engl J Med 353: 2285-2287, 2005.

6. Cantin AM, Hubbard RC and Crystal RG: Glutathione deficiency in the epithelial lining fluid of the lower respiratory tract in idiopathic pulmonary fibrosis. Am Rev Respir Dis 139: 370-372, 1989.

7. Teramoto S, Fukuchi Y, Uejima Y, Shu CY and Orimo H: Superoxide anion formation and glutathione metabolism of blood in patients with idiopathic pulmonary fibrosis. Biochem Mol Med 55: 66-70, 1995

8. Muramatsu Y, Sugino K, Ishida F, Tatebe J, Morita T and Homma S: Effect of inhaled $\mathrm{N}$-acetylcysteine monotherapy on lung function and redox balance in idiopathic pulmonary fibrosis. Respir Investig 54: 170-178, 2016.
9. Clinical diagnostic and treatment guidance for idiopathic interstitial pneumonia. In: Japanese Respiratory Society's Committee Formulating Diagnosis and Treatment Guideline for Diffuse Lung Diseases. Nankodo, Tokyo, pp63-65, 2004 (In Japanese).

10. Watanabe A, Kawaharada N and Higami T: Postoperative acute exacerbation of IPF after lung resection for primary lung cancer. Pulm Med 2011: 960316, 2011.

11. Sakamoto S, Homma S, Mun M, Fujii T, Kurosaki A and Yoshimura K: Acute exacerbation of idiopathic interstitial pneumonia following lung surgery in 3 of 68 consecutive patients: A retrospective study. Intern Med 50: 77-85, 2011.

12. Padley SP, Jordan SJ, Goldstraw P, Wells AU and Hansell DM: Asymmetric ARDS following pulmonary resection: CT findings initial observations. Radiology 223: 468-473, 2002.

13. Tiitto L, Bloigu R, Heiskanen U, Pääkkö P, Kinnula VL and Kaarteenaho-Wiik R: Relationship between histopathological features and the course of idiopathic pulmonary fibrosis/usual interstitial pneumonia. Thorax 61: 1091-1095, 2006.

14. Homma S, Azuma A, Taniguchi H, Ogura T, Mochiduki Y, Sugiyama Y, Nakata K, Yoshimura K, Takeuchi $M$ and Kudoh S; Japan NAC Clinical Study Group: Efficacy of inhaled $\mathrm{N}$-acetylcysteine monotherapy in patients with early stage idiopathic pulmonary fibrosis. Respirology 17: 467-477, 2012. 